\title{
Editorial
}

\section{Frequency of Intravenous Administration Set Changes and Bacteremia: Defining the Risk}

Robert R. Muder, MD

Despite numerous technological advances in the delivery of intravenous (IV) therapy over the past 3 decades, infection remains a major cause of morbidity and mortality among its recipients. Furthermore, IV-therapy-related infection is the single leading cause of nosocomial bacteremia. Approximately $90 \%$ of IV-therapy-related bacteremias are associated with catheter colonization. Most infections of this type are caused by gram-positive cocci, in particular staphylococci. Appropriately, most recent clinical investigations aimed at reducing the risk of IV-related infection are centered on reducing colonization of the catheter or surrounding skin by these organisms. ${ }^{1}$ Bacteremia caused by the infusion of contaminated fluids is less common, but nevertheless is a potentially serious problem that can affect single patients or dozens of patients, depending on the circumstances of contamination. Unlike catheter-related bacteremia, bacteremia due to contaminated infusate is often due to gram-negative bacilli such as Klebsiella, Enterobacter, and Serratia species, which, unlike staphylococci, can multiply in glucose-containing IV solutions. Staphylococci can, however, multiply in blood products and lipid emulsions.

Contamination of the infusate can occur through several routes. Intrinsic contamination occurs at the time of manufacture of the fluid. This type of contamination fortunately is rare, but has the potential to cause a large number of episodes of infection. In what is perhaps the best studied outbreak due to intrinsic contamination, 378 cases of bacteremia due to Enterobacter aerogenes or Enterobacter agglomerans were documented in 25 different US hospitals. ${ }^{2}$ The cases were traced to contamination of the cap used in IV-fluid bottles; bacteria were readily transferred from the cap to the fluid during manipulation of the bottle. The low numbers of bacteria present were able to multiply within the fluid; there was an association between the occurrence of bacteremia and duration of time that the bottle and administration set were in use. The clinical features of bacteremia in this outbreak were notable in that patients often had no conditions predisposing to gram-negative bacteremia; the mortality rate was $13.4 \%$. The outbreak was terminated by discontinuing use of the implicated product. More common, and perhaps more insidious, is extrinsic contamination, which occurs during subsequent admixture of IV fluids or during manipulation of the infusion apparatus. Breaks in sterile technique can introduce organisms into the infusate, where they may multiply to high concentrations. Repeated manipulation of infusion sets is a potential means of introduction of bacteria into IV fluids. ${ }^{3}$ Prolonged "hang time" of the fluid or components of the administration set may permit the multiplication of these organisms to relatively high concentration. Infusion of such fluids can lead to the abrupt onset of fever and hypotension in a previously stable patient. ${ }^{4}$ Because single patients or a very limited number of patients may be affected, such contamination may be difficult to recognize.

The Centers for Disease Control and Prevention has developed a comprehensive set of guidelines for the prevention of intravascular-device-related infections. ${ }^{5}$ With regard to replacement of administration sets, the current recommendation is that replacement should occur "... no more frequently than at 72-hour intervals. ..." An exception is made for administration of blood or lipid emulsion, which requires change of the administration set at 24 hours.

The current recommendations evolved over a 25 year period, beginning with an initial recommendation of change at 24 hours that was based on observations made during the nationwide epidemic of Enterobacter septicemia. Subsequent studies showed that changes at 48 hours $^{6-8}$ and then 72 hours $^{9}$ were not associated with an increased risk of infection. The impetus for increasing the interval is, of

From the Veterans Affairs Pittsburgh Healthcare System and the University of Pittsburgh School of Medicine, Pittsburgh, Pennsylvania. 
course, cost, which includes acquisition cost of the set and nursing time required for routine changes.

In the current issue, Raad and colleagues report the results of a randomized trial of prolonging the duration of administration set use beyond 72 hours. ${ }^{10}$ Oncology patients receiving infusion therapy were randomized to receive scheduled administration-set changes within 72 hours or at 4 to 7 days. The authors found no cases of infusion-related bacteremia, defined as isolation of the same organism from the blood and from the infusate, among 280 patients randomized to receive 72-hour changes. There were three episodes of infusion-related bacteremia among 232 patients receiving 4- to 7-day administration changes. Of note, all three episodes occurred in patients receiving interleukin-2, an oncological therapy associated with a high rate of nosocomial bacteremia. After excluding a high-risk subset of patients, defined as those receiving interleukin-2, blood transfusion, or parenteral nutrition, there were no bacteremias in either experimental group.

Although there was a somewhat higher level of bacterial contamination of infusate in the 4- to 7-day group, all bacteria isolated from infusate not associated with infection consisted of coagulase-negative staphylococci.

The study has a number of strengths in its design and execution, including careful follow-up, a high degree of comparability between experimental groups, and careful attention to microbiological methods. However, the results cannot be used to settle the question of the maximum safe interval for administration set changes definitively. The high-risk and low-risk patient subsets were designated retrospectively after the study was stopped at $75 \%$ of projected enrollment (due to the occurrence of bacteremia in the 4- to 7-day group). The sample size of the low-risk group gave insufficient power to determine comparability of the two regimens. Furthermore, $48 \%$ of administration set changes in the 4- to 7-day group occurred on day 4; there was a decreasing frequency of change at each subsequent day, with only $11 \%$ of patients receiving changes on day 7 . Thus, it is premature to conclude that extending the interval of IV access sets to 7 days is without additional risk.

There is an additional reason why one should take a conservative position on the extension of IV administration set change intervals. The impetus to extend the interval is not to improve patient care but to provide financial benefit to the institution. While the latter is certainly a worthy undertaking, it is not acceptable to increase patients' risk of serious adverse events to do so. A small number of additional bacteremias may be easily missed by routine surveillance, since infusion-related bacteremias typically represent only a small percentage of bloodstream infections. Furthermore, as the cost of an episode of IV-related bacteremia may be as high as $\$ 40,000,{ }^{11}$ the anticipated savings may fail to materialize if the rate of infection increases. Thus, I believe that the changing of IV administration sets every 4 to 7 days should be considered comparable with the current practice of change at 72 hours only if the respective risk estimates are demonstrated to lie within a fairly narrow confidence interval.

The authors are appropriately conservative in calling for a randomized, multicenter trial to confirm their observations. In addition to providing the necessary power to assure equivalency of a longer IV administration set change interval, such a study would have the merit of assessing it in diverse institutions and patient populations. Standards of IV care in an academically affiliated oncology center with a long history of research into nosocomial infection may be more meticulous than that provided in other facilities.

Evaluation of changes in practice aimed at reducing healthcare expenditure is an appropriate area of clinical investigation. Dollars available for healthcare are not limitless; money saved by eliminating unneeded equipment changes can and should be diverted into expenditures that actually benefit patients. However, since individual patients cannot expect any benefit from such trials, protection of patient safety is essential. The trial reported by Raad and colleagues in this issue offers an example of how such trials can be conducted in a responsible manner. They used an incremental approach to the intervention that was based on the results of previous trials. Informed consent was obtained from all participants. In addition, they provided a mechanism for interim analysis that could terminate the study early if a trend toward increased adverse events was noted in the experimental group. Although the authors were not able to settle the question they set out to answer definitively, their report should serve as a model for future investigations.

\section{REFERENCES}

1. Mermel LA. Prevention of intravascular catheter-related infections. Ann Intern Med 2000;132:391-402.

2. Maki DG, Rhame FS, Mackel DC, Bennet JV. Nationwide epidemic of septicemia caused by contaminated intravenous products, I: epidemiologic and clinical features. Am J Med 1976;60:471-485.

3. Duma RJ, Warner JF, Dalton HP. Septicemia from intravenous infusions. N Engl J Med 1971;284:257-260.

4. Goetz AM, Rihs JD, Chow JW, Singh N, Muder RR. An outbreak of infusion-related Klebsiella pneumoniae bacteremia in a liver transplantation unit. Clin Infect Dis 1995;21:1501-1503.

5. Pearson ML. Guideline for prevention of intravascular-device-related infections. Hospital Infection Control Practices Advisory Committee. Infect Control Hosp Epidemiol 1996;17:438-473.

6. Buxton AE, Highsmith AK, Garner JS, West CM, Stamm WE, Dixon RE, et al. Contamination of intravenous infusion fluid: effects of changing administration sets. Ann Intern Med 1979;90:764-768.

7. Gorbea HF, Snydman DR, Delaney A, Stockman J, Martin WJ. Intravenous tubing with burettes can be safely changed at 48-hour intervals. JAMA 1984;251:2112-2115.

8. Band JD, Maki DG. Safety of changing intravenous delivery systems at longer than 24-hour intervals. Ann Intern Med 1979;91:173-178.

9. Maki DG, Botticelli JT, LeRoy ML, Thielke TS. Prospective study of replacing administration sets for intravenous therapy at 48 vs 72 -hour intervals. 72 hours is safe and cost-effective. JAMA 1987;258:1777-1781.

10. Raad I, Hanna HA, Awad A, Alrahwan A, Bivins C, Khan A, et al. Optimal frequency of changing intravenous administration sets: is it safe to prolong use beyond 72 hours? Infect Control Hosp Epidemiol 2001;22:136139.

11. Pittet D, Tarara D, Wenzel RP. Nosocomial bloodstream infection in critically ill patients. Excess length of stay, extra costs, and attributable mortality. JAMA 1994;271:1598-1601. 